\section{BRAZIULIAN JOURNAL}

OF MEDICAL AND BIOLOGICAL RESHARCH

www.bjournal.com.br
ISSN 0100-879X

Volume 43 (10) 914-1009 October 2010

BIOMEDICAL SCIENCES

AND

CLINICAL INVESTIGATION

Braz J Med Biol Res, October 2010, Volume 43(10) 969-975

doi: 10.1590/S0100-879X2010007500105

Ovariectomy does not affect the cardiac sympathovagal balance of female SHR but estradiol does

D.P.M. Dias, M. Oliveira, H.C. Salgado and R. Fazan Jr.

The Brazilian Journal of Medical and Biological Research is partially financed by
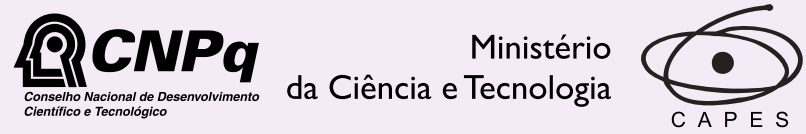

Ministério da Educação

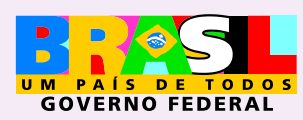

DFAPESP

Institutional Sponsors
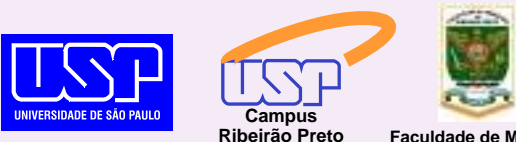

Ф SHIMADZU

GE Healthcare
Hotsite of proteomics metabolomics developped by: 


\title{
Ovariectomy does not affect the cardiac sympathovagal balance of female SHR but estradiol does
}

\author{
D.P.M. Dias, M. Oliveira, H.C. Salgado and R. Fazan Jr. \\ Departamento de Fisiologia, Faculdade de Medicina de Ribeirão Preto, \\ Universidade de São Paulo, Ribeirão Preto, SP, Brasil
}

\begin{abstract}
The low incidence of cardiovascular diseases, including hypertension, in premenopausal women has led to the conclusion that ovarian hormones may have a protective effect on the cardiovascular system. We evaluated the effects of ovariectomy and/ or estradiol on sympathovagal balance and heart rate variability (HRV) in female spontaneously hypertensive rats (SHR) with tachycardia and compared them to Wistar rats (12 weeks old; $\mathrm{N}=8-12)$. Ovariectomy (OVX) and/or estradiol (10 $\mu \mathrm{g} / \mathrm{kg}) \mathrm{did}$ not affect basal arterial pressure in either rat strain, but estradiol increased basal heart rate (HR) in OVX SHR (454 \pm 18 vs $377 \pm 9$ $\mathrm{bpm})$. HR changes elicited by methylatropine and propranolol were used to evaluate the sympathovagal balance. Ovariectomy did not affect the cardiac sympathovagal balance of any group, while estradiol increased sympathetic tone in OVX SHR (120 \pm 8 vs $56 \pm 10 \mathrm{bpm}$ ) and sham-operated Wistar rats ( $57 \pm 7$ vs $28 \pm 4 \mathrm{bpm})$, and decreased the parasympathetic tone only in OVX SHR ( $26 \pm 7$ vs $37 \pm 5 \mathrm{bpm})$. HRV was studied in the frequency domain (Fast Fourier Transformation). Spectra of HR series were examined at low frequency (LF: $0.2-0.75 \mathrm{~Hz}$ ) and high frequency (HF: $0.75-3 \mathrm{~Hz}$ ) bands. The power of LF, as well as the LF/HF ratio, was not affected by ovariectomy, but estradiol increased both LF (29 \pm 4 vs $18 \pm 3$ nu in Wistar sham-operated, $26 \pm 5$ vs $15 \pm 3$ nu in Wistar OVX, $50 \pm 3$ vs $38 \pm 4$ nu in SHR sham-operated, and $51 \pm 3$ vs $42 \pm 3$ nu in SHR OVX) and LF/ HF $(0.48 \pm 0.08$ vs $0.23 \pm 0.03$ nu in Wistar sham-operated, $0.41 \pm 0.14$ vs $0.19 \pm 0.05$ nu in Wistar OVX, $0.98 \pm 0.11$ vs 0.63 $\pm 0.11 \mathrm{nu}$ in SHR sham-operated, and $1.10 \pm 0.11$ vs $0.78 \pm 0.1 \mathrm{nu}$ in SHR OVX). Thus, we suggest that ovariectomy did not affect the cardiac sympathovagal balance of SHR or Wistar rats, while estradiol increased the sympathetic modulation of HR.
\end{abstract}

Key words: Estrogen; SHR; Heart rate variability; Hypertension; Sympathovagal balance

\section{Introduction}

Epidemiological studies indicate a low incidence of cardiovascular diseases, including hypertension, in premenopausal women compared to age-matched men (1). However, after the onset of menopause, when cardiovascular disorders become a prominent cause of death, this difference is no longer demonstrable (2). These data strongly suggest that ovarian hormones play a protective role in the cardiovascular system of young women (1). However, the mechanisms of this protective effect are unknown. Clinical and experimental investigations have suggested the existence of gender-related differences in the autonomic control of the cardiovascular system (3). There is evidence that women exhibit greater parasympathetic control of cardiovascular function compared to men (4). However, the relationship between estrogen levels and the autonomic control of the cardiovascular system is also unknown and deserves further study.

The increased incidence of cardiovascular risk in postmenopausal women may also involve changes in arterial blood pressure (BP) and its regulation, which is associated with estrogen loss. For instance, the incidence of arterial hypertension rises markedly following menopause (5). Furthermore, since arterial hypertension is a ubiquitous cardiovascular disorder and is relatively common among women during the menopausal period (5), it has been accepted that the postmenopausal reduction of estrogen levels is quite disturbing to the cardiovascular system. However, little is known about the direct effects of low estrogen levels on arterial hypertension and its autonomic regulation.

The spontaneously hypertensive rat (SHR) has been an

Correspondence: R. Fazan Jr., Departamento de Fisiologia, FMRP, USP, Av. Bandeirantes, 3900, 14049-900 Ribeirão Preto, SP, Brasil. Fax: +55-16-3633-0017. E-mail: rfazan@rfi.fmrp.usp.br

Received March 20, 2010. Accepted September 23, 2010. Available online October 8, 2010. Published October 18, 2010. 
important model for studying the effects of estrogen during the development of arterial hypertension $(6,7)$. There are a number of studies showing that female SHR have lower arterial pressure than male SHR, indicating the influence of gender on the development of hypertension $(6,8)$. Moreover, aged female SHR have been accepted as a suitable model of postmenopausal hypertension for two reasons: 1) following the cessation of cycling at approximately 11 months of age, these animals, like postmenopausal women, exhibit low estrogen levels; 2) these animals exhibit higher arterial pressure levels compared to young female SHR (6). The literature indicates that SHR have greater sympathetic tone under basal conditions compared to Wistar rats (9). Sympathetic attenuation following estrogen administration has also been demonstrated in normotensive male and female rats (10).

Heart rate variability (HRV) has been widely used to evaluate the autonomic modulation of the cardiovascular system in both humans (11) and experimental animals (12). HRV can be examined in the time domain (overall variability), as well as in the frequency domain (spectral analysis). Heart rate (HR) oscillations at low frequency (LF: 0.2 to $0.75 \mathrm{~Hz}$ ) are accepted as an index of cardiac sympathetic modulation, while high frequency (HF: 0.75 to $3 \mathrm{~Hz}$ ) oscillations of HR are considered to reflect parasympathetic modulation of the heart (11). The ratio of LF to HF power has been used to evaluate the sympathovagal balance (11). Another method used to assess this balance is a double-pharmacological blockade of sympathetic and parasympathetic receptors in the sinus node (i.e., by means of atropine and propranolol) (13).

The influence of estradiol on the cardiovascular system has been investigated in a number of clinical and experimental studies and conflicting results have been observed $(14,15)$. Moreover, there are few studies regarding the effects of estradiol on the sympathovagal balance and HRV of hypertensive animals, particularly SHR.

Therefore, the present study was undertaken to determine the effects of ovariectomy, as well as the effects of estradiol replacement therapy, on BP levels, cardiac sympathovagal balance, and HRV of conscious female SHR.

\section{Material and Methods}

Experiments were performed on 12-week-old female SHR and female Wistar rats supplied by the Animal Facility of the Faculdade de Medicina de Ribeirão Preto, Universidade de São Paulo, São Paulo, SP, Brazil. Rats were housed in a temperature-controlled room $\left(21 \pm 1^{\circ} \mathrm{C}\right)$ with a 12-h light/ dark cycle with free access to water and standard chow (Nuvilab CR-1, Nuvital, Brazil). All experimental procedures were carried out according to the Guide for the Care and Use of Laboratory Animals (DHEW Publication No. (NIH) 85-23, Revised 1985; Office of Science and Health Reports, DRR/NIH, Bethesda, MD, USA) and were approved by the
Ethics Committee for Animal Research of the Faculdade de Medicina de Ribeirão Preto, Universidade de São Paulo, Ribeirão Preto, SP, Brazil (Protocol \#0076/2006).

\section{Ovariectomy and estradiol therapy}

The animals were anesthetized with tribromoethanol (250 mg/kg, ip, Sigma, USA) and submitted to bilateral ovariectomy (OVX) or sham removal of the ovaries. All animals received prophylactic antibiotic therapy following the surgical procedures. Animals were allowed one week to recover from the surgical procedures when subcutaneous injections of estradiol benzoate $(10 \mu \mathrm{g} / \mathrm{kg}$; Sigma) or corn oil (vehicle) were applied daily for 21 days.

Before the experiments, all rats underwent estrous cycle evaluation (vaginal smear) and only rats presenting at least three regular cycles were included in the study. Next, the estrous cycle phase was evaluated every day during the last 15 days of therapy (estradiol or vehicle) to confirm that intact rats treated with vehicle were cycling regularly, OVX treated with vehicle remained in diestrus, while estradioltreated rats (sham-operated or OVX) remained in constant proestrus.

\section{Experimental groups}

SHR were assigned to the following four groups: shamoperated rats treated with vehicle $(N=12)$, sham-operated rats treated with estradiol $(\mathrm{N}=8)$, OVX rats treated with vehicle $(N=10)$, and OVX rats treated with estradiol ( $N$ = 12). Wistar rats were assigned to the following four groups: sham-operated rats treated with vehicle $(\mathrm{N}=9)$, sham-operated rats treated with estradiol $(N=10)$, OVX rats treated with vehicle $(\mathrm{N}=12)$, and OVX rats treated with estradiol $(\mathrm{N}=9)$.

\section{Heart rate and arterial pressure recordings}

After 21 days of estradiol or vehicle treatment, all animals were anesthetized with tribromoethanol $(250 \mathrm{mg} / \mathrm{kg}$, ip) and implanted with subcutaneous electrocardiogram (ECG) electrodes (lead I) and a catheter (PE-10; Clay Adams, USA) into the femoral vein. The animals were then allowed to recover for $48 \mathrm{~h}$ prior to the experimental protocol, which was carried out without anesthesia. The animals were taken to the recording room at least $1 \mathrm{~h}$ before the beginning of the recordings, and a quiet environment was maintained to avoid stress. ECG electrodes were connected to a bioelectric amplifier (8811A, Hewlett Packard, USA) and the ECG was continuously sampled $(2 \mathrm{kHz})$ using an IBM/PC computer equipped with an analog-to-digital interface (CAD 12/36, Lynx Tecnologia Eletrônica, Brazil). After $30 \mathrm{~min}$ of basal ECG recording, the rats received methylatropine $(2 \mathrm{mg} / \mathrm{kg}$, iv, Sigma) followed by propranolol (4 mg/kg, iv, Sigma) 15 min later to permit the measurement of the intrinsic heart rate (iHR). The same animals were submitted to another recording period $24 \mathrm{~h}$ later with the same autonomic blockers (i.e., methylatropine and propranolol) given in the opposite 
order. The sympathetic tone was considered to be the HR response to propranolol in rats subjected to the methylatropine/propranolol sequence, while the parasympathetic tone was the HR response to methylatropine in rats subjected to the propranolol/methylatropine sequence. Therefore, both basal HR and iHR were measured twice for each rat. The averages of the two data sets of these parameters were calculated for each animal.

Following the second ECG recording, the rats had a catheter implanted into the femoral artery under tribromoethanol anesthesia. Twenty-four hours after catheterization, the arterial line was connected to a pressure transducer (Statham, P10E2, USA) and pulsatile arterial pressure was sampled $(2 \mathrm{kHz})$ for 30 min using an IBM/PC equipped with an analog-to-digital interface.

\section{Heart rate variability}

The spectral density of the various frequency components of HR was calculated using Fast Fourier Transformation (FFT). This analysis requires data collection at equal time intervals. Therefore, beat-by-beat HR data obtained from the first $15 \mathrm{~min}$ of the first recording period were converted to data points every $100 \mathrm{~ms}$ using a cubic spline interpolation $(10 \mathrm{~Hz})$. The interpolated series was divided into half-overlapping sequential sets of 512 data points (51.2 s). Before calculation of the spectral power density, the segments were inspected visually and nonstationary data were not taken into consideration. A Hanning window was used to attenuate side effects and the spectrum was computed using a direct FFT algorithm for discrete time series. The spectra were integrated in the LF band $(0.2-0.75 \mathrm{~Hz})$ and HF band $(0.75-3$ $\mathrm{Hz}$ ), and spectral components are presented in a normalized form by dividing them by the total power minus the DC component (16), since the very low frequency (VLF) band was removed. The LF/HF ratio is also shown, defined by the LF power divided by the HF power, characterizing the sympathovagal balance $(11,16)$.

\section{Statistical analysis}

The effects of factor such as strain (Wistar rats or SHR), surgery (ovariectomy or sham operation) and treatment (estradiol or vehicle) on basal $\mathrm{HR}$, arterial pressure, vagal and sympathetic tone, iHR, as well as the HRV data were tested by three-way analysis of variance. When the effect on any factor, as well as the interactions among them, was found to be significant all pairwise multiple comparisons were performed by the StudentNewman-Keuls post hoc test. Differences were considered to be statistically significant if $\mathrm{P}<0.05$ post-test).

\section{Results}

\section{Basal arterial pressure}

Basal levels of systolic, diastolic and mean BP are shown in Table 1. As expected, BP was higher in SHR than in Wistar rats, while ovariectomy and/or estradiol did not affect these basal levels.

\section{Autonomic control of heart rate}

Figure 1 shows basal HR (dotted horizontal lines), HR after the administration of each autonomic receptor blocker, as well as the iHR (solid horizontal lines) of all groups of rats studied, i.e., SHR (right panel) and Wistar rats (left panel) submitted to ovariectomy or sham-operation, treated with estradiol or vehicle. As shown in Figure 1, SHR exhibited greater basal HR, greater cardiac sympathetic tone, as well as smaller cardiac vagal tone compared to Wistar rats. Basal HR, cardiac sympathetic tone, as well as iHR were found to be similar in OVX and sham-operated rats. Nevertheless, the significant surgery $x$ strain interaction for these variables can be interpreted as an indication that ovariectomy did exert an effect on basal HR and on the $\mathrm{HR}$ response to atropine in a rat strain-dependent manner. However, treatment with estradiol increased both basal HR and sympathetic tone, while it decreased cardiac vagal tone in OVX SHR. In contrast, estradiol did not affect these parameters in sham-operated SHR. Figure 1 also shows that estradiol increased sympathetic tone in sham-operated, but not in OVX Wistar rats.

Finally, the iHR was not affected by ovariectomy and/

Table 1. Effect of estradiol on systolic (SAP), mean (MAP) and diastolic (DAP) arterial pressures $(\mathrm{mmHg})$ of sham-operated and ovariectomized female spontaneously hypertensive rats (SHR) and Wistar rats.

\begin{tabular}{lrrrrr}
\hline & \multicolumn{2}{c}{ Sham-operated } & & \multicolumn{2}{c}{ Ovariectomized } \\
\cline { 2 - 2 } & Vehicle & Estradiol & & Vehicle & Estradiol \\
$\begin{array}{l}\text { Spontaneously hypertensive rat } \\
\text { SAP }\end{array}$ & $183 \pm 6$ & $183 \pm 6$ & & $173 \pm 5$ & $164 \pm 5$ \\
MAP & $151 \pm 6$ & $155 \pm 5$ & & $145 \pm 5$ & $139 \pm 4$ \\
DAP & $121 \pm 7$ & $129 \pm 5$ & & $117 \pm 5$ & $115 \pm 4$ \\
Wistar rat & & & & & \\
SAP & $122 \pm 5^{*}$ & $120 \pm 2^{*}$ & & $118 \pm 2^{*}$ & $123 \pm 5^{*}$ \\
DAP & $99 \pm 4^{*}$ & $95 \pm 2^{*}$ & & $97 \pm 2^{*}$ & $99 \pm 5^{*}$ \\
MAP & $83 \pm 4^{*}$ & $79 \pm 1^{*}$ & & $81 \pm 2^{*}$ & $82 \pm 4^{*}$ \\
\hline
\end{tabular}

Data are reported as means \pm SEM. Three-way ANOVA showed a strain effect (Wistar vs SHR) for basal systolic, mean and diastolic arterial blood pressures $(P<0.001)$. No significant effects of surgery (sham operation vs ovariectomy) or treatment (vehicle vs estrogen) on arterial pressure were detected. Interactions between strain and treatment and between surgery and treatment were also not statistically significant. ${ }^{*} P<0.05$ compared to SHR (Student-Newman-Keuls 


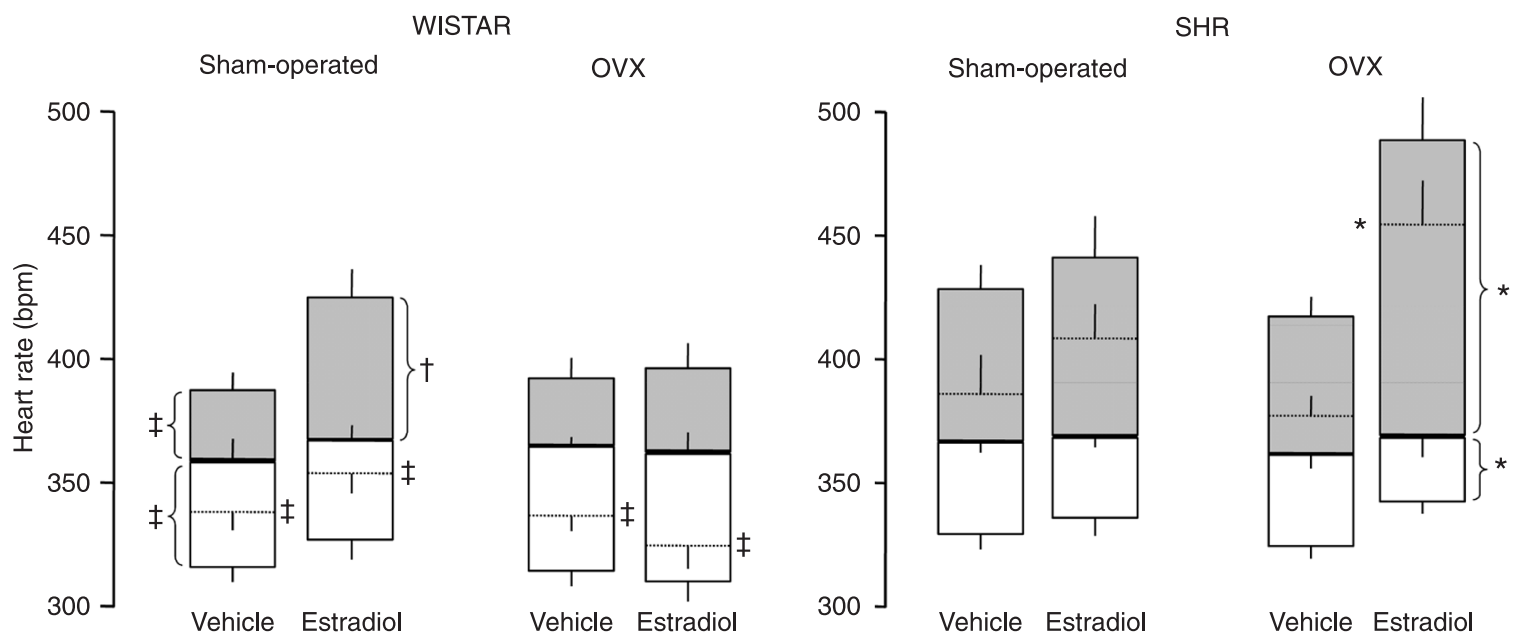

Figure 1. Effect of estradiol on cardiac sympathetic and vagal tones. Bar graphs showing basal heart rate (dotted horizontal line), intrinsic heart rate (solid horizontal line) and heart rate responses to propranolol after methyl-atropine (cardiac sympathetic tone: gray bars) or to methyl-atropine after propranolol (cardiac vagal tone: open bars) of sham-operated and ovariectomized (OVX) spontaneously hypertensive rats (SHR) and Wistar rats treated with vehicle or estradiol. Three-way ANOVA showed a strain effect (Wistar vs SHR) for basal HR $(P<0.001)$ and for HR response to propranolol $(P=0.003)$ and methyl-atropine $(P<0.001)$. No significant effect of surgery (sham operation vs ovariectomy) was detected on any parameter shown in the figure. Treatment effect (vehicle vs estrogen) was significant for basal HR $(P=0.003)$ and for HR response to methylatropine $(P<0.001)$. Interaction between strain and surgery was significant for basal HR $(P=0.047)$ and for $H R$ response to methylatropine $(P=0.013)$. Interaction between strain and treatment was significant only for basal $H R$ $(P=0.005)$. No differences were found for interaction between surgery and treatment for the parameters shown in the figure. ${ }^{*} P<0.05$ compared to the same parameter of OVX SHR treated with vehicle; $\mathrm{TP}_{\mathrm{P}}<0.05$ compared to the same parameter of sham-operated Wistar treated with vehicle; $¥ \mathrm{P}<0.05$ compared to the same parameter of SHR (Student-Newman-Keuls post hoc test).

or estradiol in any group of rats examined.

\section{Heart rate variability}

Figure 2 shows that pulse interval $(\mathrm{PI})$ spectra from Wistar rats presented a lower power of the LF band and a higher power of the HF band, as well as a lower LF/HF ratio, compared to SHR. Ovariectomy did not affect LF or HF power, while estradiol increased LF and decreased HF power of $\mathrm{PI}$ spectra in both rat strains. Finally, ovariectomy did not affect the LF/HF ratio, while estradiol did. When HRV was examined in the time domain, ovariectomy did not affect the cardiac PI in either rat strain. Nevertheless, estradiol did affect the PI only in SHR submitted to ovariectomy. No effect of ovariectomy and/or estradiol was observed on PI variance in either rat strain.

\section{Discussion}

Despite the evidence from the literature that the lack of estrogen can contribute to sympathoexcitation, as well as increasing arterial pressure, our results did not show any conspicuous effect of ovariectomy on basal BP and HR levels of female SHR or Wistar rats. Moreover, estrogen treatment not only failed to decrease the sympathetic tone, but rather increased it.

The estrogen levels achieved with chronic estradiol therapy were not measured in the present study. Although it is recognized that different regimens of estradiol administration may produce different hormone level profiles (17), a therapy similar to that used in the present study led to estradiol levels comparable to those observed during proestrous (18).

As reported in previous studies, ovariectomy promotes a marked reduction of estrogen levels (19). Nevertheless, in our study, ovariectomy did not affect cardiac autonomic tone. One may hypothesize that, after removing the ovaries, a number of other forms of estrogen may have been increased by peripheral hormone synthesis (19), explaining why ovariectomy failed to demonstrate any effect on autonomic tone. In the present study, ovariectomy was performed in young adult female rats, a different model from menopausal estrogen depletion (20).

\section{Basal arterial pressure}

The hypertensive levels exhibited by female SHR compared to female Wistar rats agreed with previous data from the literature (21) obtained in the same time frame as examined in the present study. In the current study, ovariectomy and/or estradiol did not affect basal BP of female SHR or Wistar rats. There is no consensus in the literature regarding the effects of ovariectomy and/or estradiol therapy on BP levels.

Previous studies have indicated that ovariectomy did not affect BP of conscious (22) or anesthetized (23), hyper- 
tensive or normotensive female rats. There are, however, studies showing that hypertensive and normotensive rats submitted to ovariectomy exhibited an increase in BP (24), which was reversed (24), or not (22), by chronic estradiol therapy. It is likely that this lack of consensus is due to either the different methods used for BP recording or to a modulatory role of estrogen on the central nervous system (CNS) and/or peripheral organs, whose effect is not yet well understood. Nevertheless, the results of the current study indicate, by means of direct recording of the BP without the undesirable effect of anesthesia, that ovariectomy and/or estradiol did not affect the BP of female SHR or Wistar rats.

\section{Autonomic control of heart rate}

Ovariectomy did not affect basal HR, sympathovagal balance, or iHR in female SHR and Wistar rats. However, female sham-operated Wistar rats showed greater cardiac sympathetic tone following chronic estradiol administration. This therapy also affected the female SHR submitted to ovariectomy, increasing basal HR and sympathetic tone and decreasing parasympathetic tone. Thus, the results of the present study demonstrate that estrogen therapy causes an imbalance in the autonomic control of HR, displacing the equilibrium toward increased sympathetic activity. Since iHR did not differ among groups, this finding indicates that ovariectomy and/or estradiol most likely did not act directly on the sinus node. It is likely that estradiol affects the sympathetic tone by means of an action on the CNS, since there are estrogen receptors in areas of the CNS related to cardiovascular control, such as preoptic area, paraventricular nucleus and nucleus of the tractus solitarii $(25,26)$.

There is, however, evidence that estradiol administration directly into nuclei related to cardiovascular control decreases sympathetic and increases parasympathetic activity in male Sprague Dawley rats (27). Experiments carried out in vitro have shown that supra-physiological estrogen levels decrease tyrosine hydroxylase (a rate-limiting enzyme in catecholamine biosynthesis) production (28), while ovariectomy reduces acetylcholine synthesis in the CNS, which is restored by chronic estrogen therapy (29).

\section{Heart rate variability}

$\mathrm{HRV}$ is used as an important index of cardiac autonomic modulation and is usually taken as a parameter of cardiovascular health $(11,16)$. Analysis of HRV in the frequency domain (spectral analysis) allows the evaluation of cardiac autonomic modulation. The power of the LF band of HR spectra is widely accepted as an index of sympathetic modulation, while the power of the HF band is associated with parasympathetic modulation (11). Nevertheless, there is evidence that the LF power of HR spectra also represents parasympathetic modulation (30). Thus, the power of LF and HF bands represent more accurately the autonomic
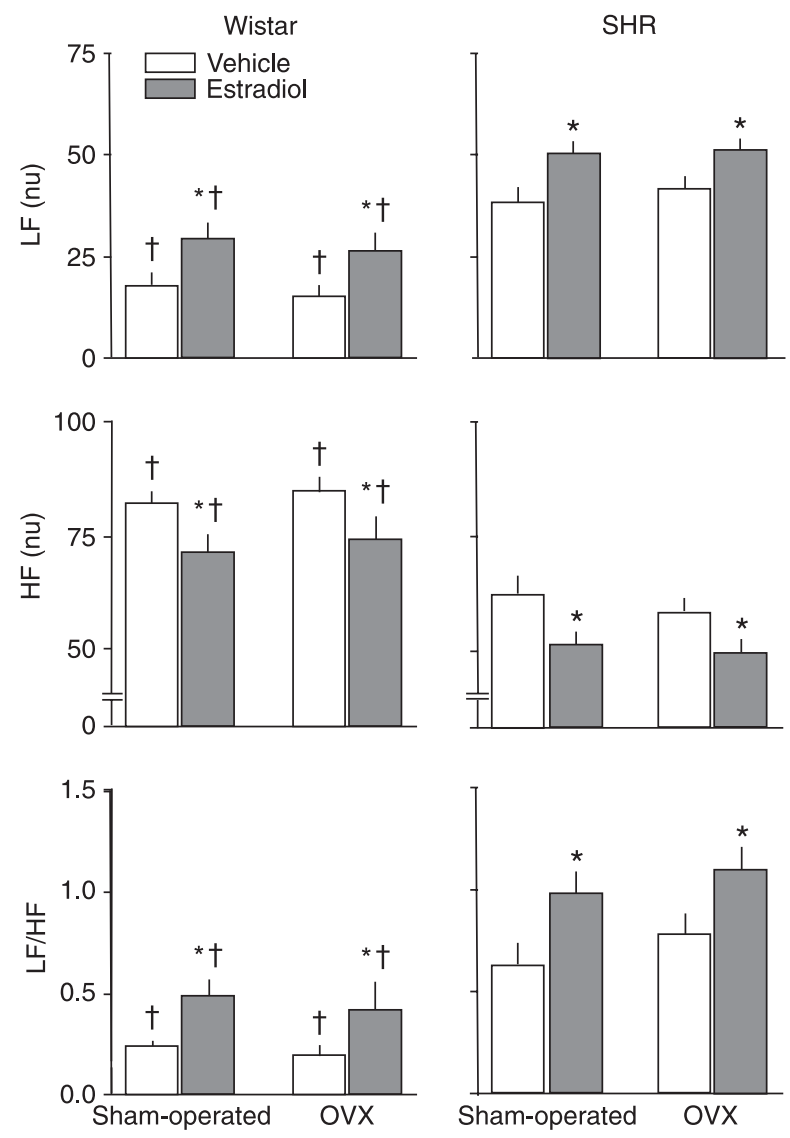

Figure 2. Upper panel, Power of HR spectra at the low frequency (LF) band (normalized units; nu) for sham-operated and ovariectomized (OVX) spontaneously hypertensive rats (SHR) and Wistar rats treated with vehicle or estradiol. Middle panel, Power of HR spectra at the high frequency (HF) band from sham-operated and OVX SHR and Wistar rats treated with vehicle or estradiol. Lower panel, Ratio between the power of HR spectra at the LF and HF bands for sham-operated and OVX female SHR and Wistar rats treated with vehicle or estradiol. Three-way ANOVA showed a significant effect of both strain (Wistar vs SHR) and treatment (vehicle vs estrogen) for LF, HF and LF/HF $(P<0.001)$. No significant effect of surgery (sham operation or ovariectomy) was detected on any parameter shown in the figure. Interactions between strain and surgery, strain and treatment or surgery and treatment were not significant. ${ }^{*} \mathrm{P}<0.05$ compared to vehicle; tP $<0.05$ compared to SHR (Student-Newman-Keuls post hoc test).

modulation when presented in normalized units, or by means of the LF/HF ratio (16). The results of the present study showed that ovariectomy did not affect HR spectra or the LF/HF ratio of the HR spectra of female SHR and Wistar rats. However, the power of LF was increased while the power of HF was decreased under estradiol, leading to an increased LF/HF ratio in both rat strains studied. These data suggest that estradiol treatment elicited autonomic imbalance, with sympathetic predominance, in female rats 
of both strains, suggesting that the preexisting autonomic imbalance of SHR did not play a major role in the effect of estrogen treatment in the animals studied.

A number of studies have indicated that acute systemic administration of estrogen decreases sympathetic and increases parasympathetic activity in female and male Sprague Dawley rats $(10,31)$. Moreover, the sympathetic overactivity observed following middle cerebral artery occlusion is attenuated by previous estrogen injection into the parabrachial nucleus of male Sprague Dawley rats (32). In addition, when an estrogen antagonist is previously injected into the nucleus tractus solitarii of male Sprague Dawley rats, the estrogen-mediated recovery of autonomic function following middle cerebral artery occlusion is attenuated, supporting the importance of estrogen receptor availability within the CNS (27).

Nevertheless, a careful examination of the literature revealed conflicting results regarding the effects of estradiol therapy on the autonomic tone of women. In agreement with our findings is the observation that during a regular menstrual cycle when estradiol concentration is high, there is an increase in sympathetic activity with no change in arterial pressure (33). Moreover, direct recordings from peripheral sympathetic nerves revealed increased sympathetic outflow during pregnancy in women (34) as well as an increase in tyrosine hydroxylase in adrenal glands and superior cervical ganglia during pregnancy as compared to diestrus and ovariectomy (35).

\section{References}

1. Becker RC, Corrao JM. Cardiovascular disease in women: scope of the problem. Cardiology 1990; 77 (Suppl 2): 6-7.

2. Mondul AM, Rodriguez C, Jacobs EJ, Calle EE. Age at natural menopause and cause-specific mortality. Am J Epidemiol 2005; 162: 1089-1097.

3. Dart AM, Du XJ, Kingwell BA. Gender, sex hormones and autonomic nervous control of the cardiovascular system. Cardiovasc Res 2002; 53: 678-687.

4. Ryan SM, Goldberger AL, Pincus SM, Mietus J, Lipsitz LA. Gender- and age-related differences in heart rate dynamics: are women more complex than men? J Am Coll Cardiol 1994; 24: 1700-1707.

5. Dubey RK, Oparil S, Imthurn B, Jackson EK. Sex hormones and hypertension. Cardiovasc Res 2002; 53: 688-708.

6. Fortepiani LA, Zhang H, Racusen L, Roberts LJ, Reckelhoff JF. Characterization of an animal model of postmenopausal hypertension in spontaneously hypertensive rats. Hypertension 2003; 41: 640-645.

7. van Eickels M, Schreckenberg R, Doevendans PA, Meyer R, Grohe C, Schluter KD. The influence of oestrogen-deficiency and ACE inhibition on the progression of myocardial hypertrophy in spontaneously hypertensive rats. Eur J Heart Fail 2005; 7: 1079-1084.

8. Reckelhoff JF, Zhang H, Granger JP. Testosterone exacerbates hypertension and reduces pressure-natriuresis in
A hypothesis to explain the high sympathetic activity following estradiol therapy could take into account the effect of this hormone on nitric oxide (NO), since NO production is increased by estrogen (36). Thus, the increase in sympathetic activity might be explained as a compensatory mechanism for the reduced vascular tone elicited by NO, such as the sympathetic activation elicited by the fall in pressure due to nifedipine treatment in hypertensive patients (37). Nevertheless, the mechanisms responsible for the sympatho-excitation found in rats treated with estrogen remains to be elucidated.

The findings of the present study indicate that ovariectomy did not affect the cardiac sympathovagal balance in either rat strain, while estradiol increased the cardiac sympathovagal balance of female SHR and Wistar rats. Despite the evidence that hormone replacement therapy may positively affect the cardiovascular system reducing the risk of cardiovascular disturbances $(38,39)$, the results of the present study agree with clinical evidence (40) indicating that estradiol therapy may not affect, or may negatively affect, the cardiovascular system, since after estrogen therapy the cardiovascular risk may not be reduced and the probability of cardiovascular derangements may be increased in women.

\section{Acknowledgments}

Research supported by FAPESP, CAPES and CNPq. male spontaneously hypertensive rats. Hypertension 1998; 31: 435-439.

9. Lundin S, Ricksten SE, Thoren P. Renal sympathetic activity in spontaneously hypertensive rats and normotensive controls, as studied by three different methods. Acta Physiol Scand 1984; 120: 265-272.

10. Saleh TM, Connell BJ, Saleh MC. Acute injection of 17 betaestradiol enhances cardiovascular reflexes and autonomic tone in ovariectomized female rats. Auton Neurosci 2000; 84: 78-88.

11. Heart rate variability. Standards of measurement, physiological interpretation, and clinical use. Task Force of the European Society of Cardiology and the North American Society of Pacing and Electrophysiology. Eur Heart $J$ 1996; 17: 354-381.

12. Fazan R Jr, Dias da Silva V, Ballejo G, Salgado HC. Power spectra of arterial pressure and heart rate in streptozotocininduced diabetes in rats. $J$ Hypertens 1999; 17: 489-495.

13. Jose AD, Taylor RR. Autonomic blockade by propranolol and atropine to study intrinsic myocardial function in man. J Clin Invest 1969; 48: 2019-2031.

14. Hulley S, Grady D, Bush T, Furberg C, Herrington D, Riggs $B$, et al. Randomized trial of estrogen plus progestin for secondary prevention of coronary heart disease in postmenopausal women. Heart and Estrogen/Progestin 
Replacement Study (HERS) Research Group. JAMA 1998; 280: 605-613.

15. Vongpatanasin $\mathrm{W}$, Tuncel M, Mansour Y, Arbique D, Victor RG. Transdermal estrogen replacement therapy decreases sympathetic activity in postmenopausal women. Circulation 2001; 103: 2903-2908.

16. Montano N, Ruscone TG, Porta A, Lombardi F, Pagani M, Malliani A. Power spectrum analysis of heart rate variability to assess the changes in sympathovagal balance during graded orthostatic tilt. Circulation 1994; 90: 1826-1831.

17. Strom JO, Theodorsson E, Holm L, Theodorsson A. Different methods for administering 17beta-estradiol to ovariectomized rats result in opposite effects on ischemic brain damage. BMC Neurosci 2010; 11: 39.

18. Yang X, Liu R, Dong Y. Regulative effects of ovarian steroids on rat gastric motility and sensitivity. Sheng Li Xue Bao 2006; 58: $275-280$.

19. Davidge ST, Zhang Y, Stewart KG. A comparison of ovariectomy models for estrogen studies. Am J Physiol Regul Integr Comp Physiol 2001; 280: R904-R907.

20. Clark JT, Chakraborty-Chatterjee M, Hamblin M, Wyss JM, Fentie $\mathrm{IH}$. Estrogen depletion differentially affects blood pressure depending on age in Long-Evans rats. Endocrine 2004; 25: 173-186.

21. Kauser K, Rubanyi GM. Gender difference in endothelial dysfunction in the aorta of spontaneously hypertensive rats. Hypertension 1995; 25: 517-523.

22. Gimenez J, Garcia MP, Serna M, Bonacasa B, Carbonell LF, Quesada T, et al. 17Beta-oestradiol enhances the acute hypotensive effect of captopril in female ovariectomized spontaneously hypertensive rats. Exp Physiol 2006; 91: 715-722.

23. Jazbutyte V, Hu K, Kruchten P, Bey E, Maier SK, Fritzemeier $\mathrm{KH}$, et al. Aging reduces the efficacy of estrogen substitution to attenuate cardiac hypertrophy in female spontaneously hypertensive rats. Hypertension 2006; 48: 579-586.

24. Dantas AP, Scivoletto R, Fortes ZB, Nigro D, Carvalho MH. Influence of female sex hormones on endothelium-derived vasoconstrictor prostanoid generation in microvessels of spontaneously hypertensive rats. Hypertension 1999; 34: 914-919.

25. Pelletier G, Liao N, Follea N, Govindan MV. Mapping of estrogen receptor-producing cells in the rat brain by in situ hybridization. Neurosci Lett 1988; 94: 23-28.

26. Stefano GB, Prevot V, Beauvillain JC, Cadet P, Fimiani C, Welters I, et al. Cell-surface estrogen receptors mediate calcium-dependent nitric oxide release in human endothelia. Circulation 2000; 101: 1594-1597.

27. Saleh TM, Cribb AE, Connell BJ. Role of estrogen in central nuclei mediating stroke-induced changes in autonomic tone. J Stroke Cerebrovasc Dis 2003; 12: 182-195.

28. Lloyd T, Weisz J. Direct inhibition of tyrosine hydroxylase activity by catechol estrogens. J Biol Chem 1978; 253: 48414843.

29. O'Malley CA, Hautamaki RD, Kelley M, Meyer EM. Effects of ovariectomy and estradiol benzoate on high affinity choline uptake, ACh synthesis, and release from rat cerebral cortical synaptosomes. Brain Res 1987; 403: 389-392.

30. Appel ML, Berger RD, Saul JP, Smith JM, Cohen RJ. Beat to beat variability in cardiovascular variables: noise or music? J Am Coll Cardiol 1989; 14: 1139-1148.

31. Saleh TM, Connell BJ. Centrally mediated effect of 17 betaestradiol on parasympathetic tone in male rats. Am J Physiol 1999; 276: R474-R481.

32. Saleh TM, Connell BJ, Cribb AE. Estrogen in the parabrachial nucleus attenuates the sympathoexcitation following MCAO in male rats. Brain Res 2005; 1066: 187-195.

33. Minson CT, Halliwill JR, Young TM, Joyner MJ. Influence of the menstrual cycle on sympathetic activity, baroreflex sensitivity, and vascular transduction in young women. Circulation 2000; 101: 862-868.

34. Greenwood JP, Scott EM, Stoker JB, Walker JJ, Mary DA. Sympathetic neural mechanisms in normal and hypertensive pregnancy in humans. Circulation 2001; 104: 2200-2204.

35. Anglin JC, Brooks VL. Tyrosine hydroxylase and norepinephrine transporter in sympathetic ganglia of female rats vary with reproductive state. Auton Neurosci 2003; 105: 8-15.

36. Rahimian R, Chan L, Goel A, Poburko D, van Breemen C. Estrogen modulation of endothelium-derived relaxing factors by human endothelial cells. Biochem Biophys Res Commun 2004; 322: 373-379.

37. Leppert J, Nilsson H, Myrdal U, Edvinsson L, Hedner T, Ringqvist I. Sympathetic activation after two weeks of nifedipine treatment in primary Raynaud's patients and controls. Cardiovasc Drugs Ther 1993; 7: 901-907.

38. Grodstein F, Stampfer MJ, Colditz GA, Willett WC, Manson JE, Joffe M, et al. Postmenopausal hormone therapy and mortality. N Engl J Med 1997; 336: 1769-1775.

39. Grodstein F, Stampfer MJ, Manson JE, Colditz GA, Willett WC, Rosner B, et al. Postmenopausal estrogen and progestin use and the risk of cardiovascular disease. N Engl J Med 1996; 335: 453-461.

40. Rossouw JE, Anderson GL, Prentice RL, LaCroix AZ, Kooperberg C, Stefanick ML, et al. Risks and benefits of estrogen plus progestin in healthy postmenopausal women: principal results From the Women's Health Initiative randomized controlled trial. JAMA 2002; 288: 321-333. 